\title{
SARAMPIÓN AMENAZA REEMERGENTE DE EPIDEMIA EN EL PERÚ
}

\author{
MEASLES: REEMERGING THREAT OF EPIDEMIC IN PERU \\ Manuel J. Loayza-Alarico ${ }^{1,2, a, c}$, Jhony A. De La Cruz -Vargas $2,3, b, d$
}

\begin{abstract}
El sarampión considerado entre las infecciones transmisibles más contagiosas por su alto riesgo potencial epidémico de transmisión, se mantiene como foco de preocupación en el mundo por el incremento de casos y su consecuente morbilidad y mortalidad'. El exantema se inicia habitualmente en la cara, y de forma centrifuga se disemina a tronco y extremidades. A pesar de que la enfermedad determina una fuerte respuesta inmune humoral $y$ celular estimulando inmunidad específica de por vida, produce a la vez una importante inmunosupresión que dura varias semanas aumentando la susceptibilidad a infecciones secundarias ${ }^{2}$.
\end{abstract}

El sarampión se consideró eliminado de las Américas desde 2002 debido a la ausencia de transmisión endémica de la enfermedad en la región; logro alcanzado por los esfuerzos regionales por lograr altas coberturas de vacunación a través de programas regulares de inmunización y campañas masivas de vacunación. Sin embargo, hasta abril del 2019 la Oficina Panamericana de la Salud informo que 12 países notificaron casos confirmados: Argentina, Bahamas, Brasil, Canadá, Chile, Colombia, Costa Rica, Estados Unidos de América, México, Perú, Uruguay y Venezuela. 09 países notificaron casos importados de países fuera de la Región con diferentes genotipos. Asimismo, entre el 1 de enero y el 11 de abril de 2019 se confirmaron 5556 casos de sarampión en 20 Estados de los Estados Unidos ${ }^{3}$.

El aumento de los viajes y el comercio internacional, así como la aparición de amenazas de transmisión del sarampión asociado a la migración poblacional de zonas en donde sus tasas de vacunación están por debajo de los niveles para prevenir la propagación (coberturas de sarampión del 95\% y que los niños reciban dos dosis de la vacuna contra el sarampión antes de su quinto año de vida) ha puesto a esta enfermedad en la prioridad de la agenda de la salud pública. Es así que en el marco del Reglamento Sanitario Internacional (RSI) se han intensificado las comunicaciones de los Centros de Enlace Nacional en la detección, seguimiento y control de casos sospechosos y confirmados de sarampión que ingresan o salen de los países con la finalidad de que se realizan intervenciones en salud pública para mitigar y disminuir el riesgo de propagación de casos que ingresan por las zonas de frontera.

Es así que el año 2018, en el Perú se confirmaron 42 casos de sarampión, importados o relacionados a importación. No se registraron fallecidos. El 26\% (11) de los casos fueron menores de 1 año. Los casos confirmados en procedieron de Amazonas, Callao, Cusco, Ica, La Libertad, Lima, Piura y Puno y las vías de ingreso de estos pacientes fueron por vía terrestre, aérea y fluvial (Figura 1). Durante el año 2019 en el Perú se confirmó un caso de sarampión que corresponde a una mujer de 40 años, peruana procedente de España que ingresó a Perú el 21 de marzo. Inició exantema el 1 de abril, fue hospitalizada por neumonía. No se detectaron casos secundarios a este caso ${ }^{3}$.

Ante este contexto, la OPS está recomendando5: (a) Realizar campañas de vacunación para mantener coberturas homogéneas del $95 \%$ con la primera y segunda dosis de la vacuna contra el sarampión, la rubéola y las paperas; (b) identificar y vacunar a poblaciones en riesgo, como personal de salud, personas que trabajan en turismo y transporte (hotelería, aeropuerto, taxis y otros); (c) identificar los flujos migratorios externos (llegada de personas extranjeras) e internos (desplazamientos de grupos poblacionales) en cada país, según el esquema nacional; (d) implementar un plan para inmunizar a la población migrante en las fronteras de alto tránsito, priorizando la población en riesgo, tanto migrante como residente en los distritos que albergan a estos grupos de población; (e) brindar una respuesta rápida frente a los casos importados de sarampión para evitar el restablecimiento de la transmisión endémica.

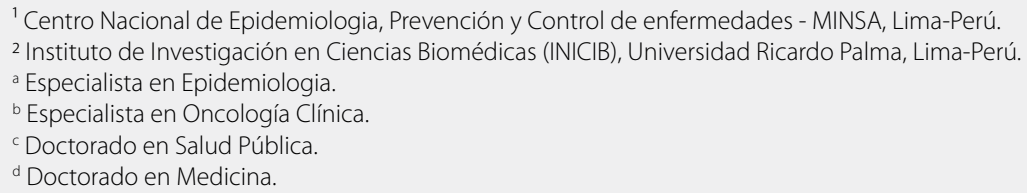

Citar como: Manuel J. Loayza-Alarico, Jhony A. De La Cruz -Vargas. Sarampión amenaza reemergente de epidemia en el Perú. Rev. Fac. Med. Hum. Julio 2019; 19(3):7-8. DOI 10.25176/RFMH.v19i3.2176 


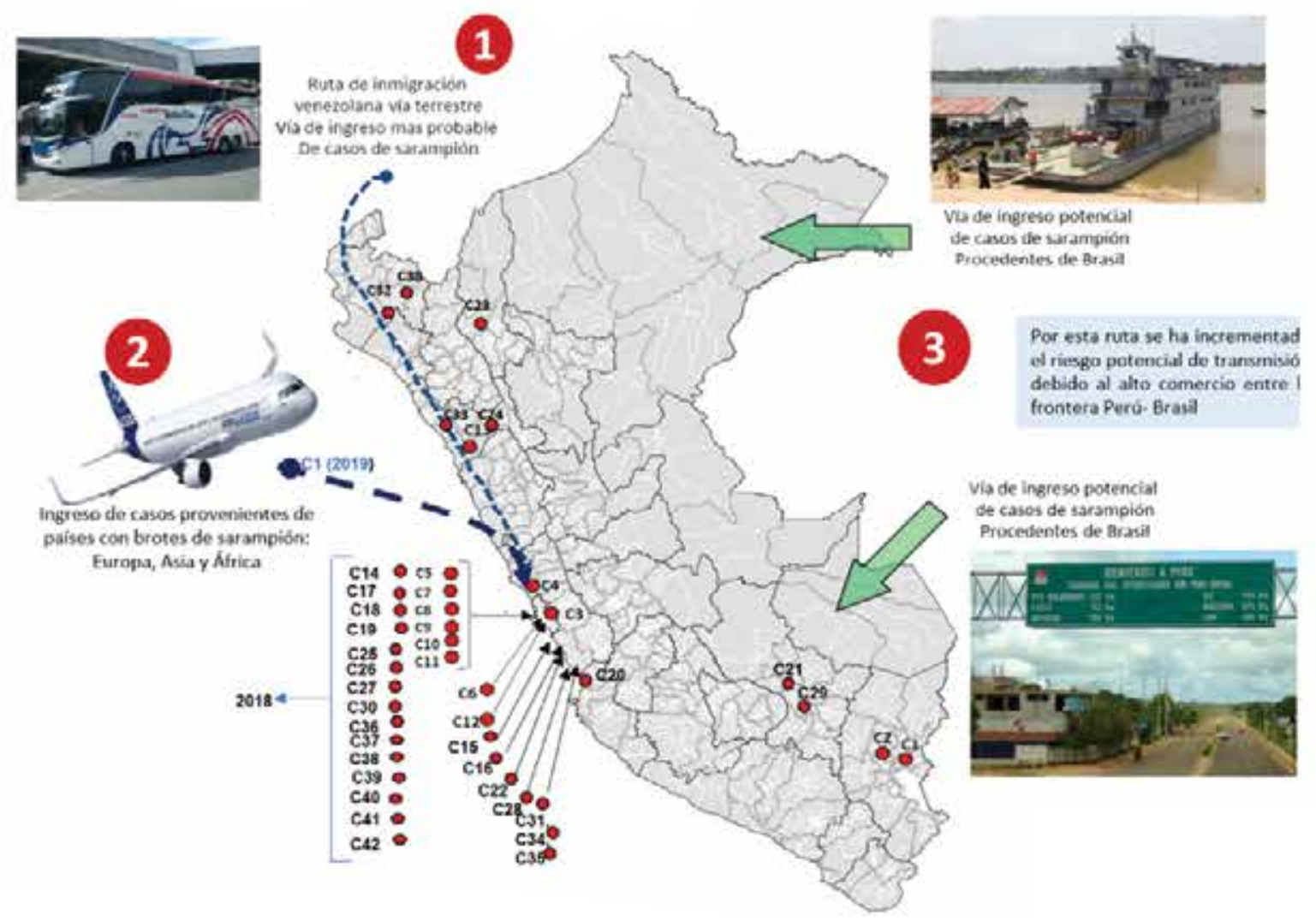

Elaborado por: Manuel Loayza Alarico-CDC-MINSA

Figura 1. Rutas de ingreso de casos de sarampión 2018-2019, Perú.

\section{REFERENCIAS BIBLIOGRÁFICAS}

1.Mamou M, Shefer A, Jankovic D, Deshevoy S, Butler R. Butler. Situación del sarampión y la rubeola en la región europea de la OMS. Rev. Esp. Salud Pública 2015;89 (4): 345-351.

2.Delpiano Luis, Astroza Leonor, Toro Jorge. Sarampión: la enfermedad, epidemiología, historia y los programas de vacunación en Chile. Rev. chil. infectol. [Internet]. 2015 Ago [citado 2019 Mayo 02] ; 32( 4 ): 417-429. Disponible en: https://scielo.conicyt.cl/scielo.php?script=sci_ arttext\&pid=S0716-10182015000500008\&lng=es.
3.Organización Panamericana de la Salud / Organización Mundial de la Salud. Actualización Epidemiológica: Sarampión. 18 de abril de 2019, Washington, D.C.: OPS/OMS;2019

4.Organización Mundial de la Salud. Reglamento Sanitario Internacional. 3ra edición. France

5.Organización Panamericana de la Salud / Organización Mundial de la Salud. Actualización Epidemiológica: Sarampión. 17 de mayo de 2019, Washington, D.C.: OPS/OMS;2019 